\title{
Convolutions Involving the Exponential Function and the Exponential Integral
}

\author{
Brian Fisher And Fatma AL-Sirehy
}

\begin{abstract}
The exponential integral ei $(\lambda x)$ and its associated functions $\mathrm{ei}_{+}(\lambda x)$ and $\mathrm{ei}-(\lambda x)$ are defined as locally summable functions on the real line and their derivatives are found as distributions. The convolutions $x^{r} \mathrm{ei}_{+}(x) * x^{s} e_{+}^{x}$ and $x^{r} \mathrm{ei}_{+}(x) * x^{s} e^{x}$ are evaluated.
\end{abstract}

\section{Introduction And Results}

The exponential integral ei $(x)$ is defined for $x>0$ by

$$
\mathrm{ei}(x)=\int_{x}^{\infty} u^{-1} e^{-u} \mathrm{~d} u
$$

see Sneddon [8], the integral diverging for $x \leq 0$. It was pointed out in [1] that equation (1) can be rewritten in the form

$$
\operatorname{ei}(x)=\int_{x}^{\infty} u^{-1}\left[e^{-u}-H(1-u)\right] \mathrm{d} u-H(1-x) \ln |x|,
$$

where $H$ denotes Heaviside's function. The integral in this equation is convergent for all $x$ and so we use equation (2) to define ei $(x)$ on the real line.

More generally, see [1], if $\lambda \neq 0$, we define ei $(\lambda x)$ in the obvious way by

$$
\operatorname{ei}(\lambda x)=\int_{\lambda x}^{\infty} u^{-1}\left[e^{-u}-H(1-u)\right] \mathrm{d} u-H(1-\lambda x) \ln |\lambda x| .
$$

Further, we define the functions $\mathrm{ei}_{+}(\lambda x)$ and $\mathrm{ei}_{-}(\lambda x)$ by

$$
\mathrm{ei}_{+}(\lambda x)=H(x) \mathrm{ei}(\lambda x), \quad \text { ei }-(\lambda x)=H(-x) \operatorname{ei}(\lambda x)
$$

so that

$$
\mathrm{ei}(\lambda x)=\mathrm{ei}_{+}(\lambda x)+\mathrm{ei}_{-}(\lambda x) .
$$

In particular, if $\lambda>0$, we have

$$
\operatorname{ei}(\lambda x)=\int_{x}^{\infty} u^{-1}\left[e^{-\lambda u}-H(1-\lambda u)\right] \mathrm{d} u-H(1-\lambda x) \ln |\lambda x|,
$$

2010 Mathematics Subject Classification. Primary: 33B10, 46F10.

Key words and phrases. exponential integral, convolution. 
(6) $\quad \mathrm{ei}_{+}(\lambda x)=\int_{x}^{\infty} u^{-1} e^{-\lambda u} \mathrm{~d} u, \quad x>0$,

$$
\mathrm{ei}_{-}(\lambda x)=-\gamma(\lambda)+\int_{x}^{0} u^{-1}\left(e^{-\lambda u}-1\right) \mathrm{d} u-\ln x_{-}, \quad x<0,
$$

where

$$
\gamma(\lambda)=\gamma+\ln |\lambda|
$$

and

$$
\gamma=-\int_{0}^{\infty} u^{-1}\left[e^{-\lambda u}-H(1-\lambda u)\right] \mathrm{d} u
$$

is Euler's constant.

The derivatives of these functions are given by

$$
\begin{aligned}
{[\mathrm{ei}(\lambda x)]^{\prime} } & =-e^{-\lambda x} x^{-1}, \\
{\left[\mathrm{ei}_{+}(\lambda x)\right]^{\prime} } & =-e^{-\lambda x} x_{+}^{-1}-\gamma(\lambda) \delta(x), \\
{\left[\mathrm{ei}_{-}(\lambda x)\right]^{\prime} } & \left.=e^{-\lambda x} x_{-}^{-1}+\gamma(\lambda) \delta(x)\right),
\end{aligned}
$$

for all $\lambda \neq 0$.

In particular, we have

$$
\begin{aligned}
\mathrm{ei}(x) & =\int_{x}^{\infty} u^{-1}\left[e^{-u}-H(1-u)\right] \mathrm{d} u-H(1-x) \ln |x|, \\
\mathrm{ei}_{+}(x) & =\int_{x}^{\infty} u^{-1} e^{-u} \mathrm{~d} u, \quad x>0, \\
\mathrm{ei}-(x) & =-\gamma+\int_{x}^{0} u^{-1}\left(e^{-u}-1\right) \mathrm{d} u-\ln x_{-}, \quad x<0,
\end{aligned}
$$

where

$$
\gamma=-\int_{0}^{\infty} u^{-1}\left[e^{-u}-H(1-u)\right] \mathrm{d} u
$$

is Euler's constant.

The derivatives of these functions are given by

$$
\begin{aligned}
{[\mathrm{ei}(x)]^{\prime} } & =-e^{-x} x^{-1}, \\
{\left[\mathrm{ei}_{+}(x)\right]^{\prime} } & =-e^{-x} x_{+}^{-1}-\gamma \delta(x), \\
{\left[\mathrm{ei}_{-}(x)\right]^{\prime} } & =e^{-x} x_{-}^{-1} .
\end{aligned}
$$

The classical definition of the convolution of two functions $f$ and $g$ is as follows:

Definition 1. Let $f$ and $g$ be functions. Then the convolution $f * g$ is defined by

$$
(f * g)(x)=\int_{-\infty}^{\infty} f(t) g(x-t) \mathrm{d} t
$$

for all points $x$ for which the integral exist. 
It follows easily from the definition that if $f * g$ exists then $g * f$ exists and

$$
f * g=g * f
$$

and if $(f * g)^{\prime}$ and $f * g^{\prime}$ (or $f^{\prime} * g$ ) exists, then

$$
(f * g)^{\prime}=f * g^{\prime}\left(\text { or } f^{\prime} * g\right) .
$$

Definition 1 can be extended to define the convolution $f * g$ of two distributions $f$ and $g$ in $D^{\prime}$ with the following definition, see Gel'fand and Shilov [7].

Definition 2. Let $f$ and $g$ be distributions in $D^{\prime}$. Then the convolution $f * g$ is defined by the equation

$$
\langle(f * g)(x), \varphi\rangle=\langle f(y),\langle g(x), \varphi(x+y)\rangle\rangle
$$

for arbitrary $\varphi$ in $D$, provided $f$ and $g$ satisfy either of the conditions

(a) either $f$ or $g$ has bounded support,

(b) the supports of $f$ and $g$ are bounded on the same side.

It follows that if the convolution $f * g$ exists by this definition then equations (10) and (11) are satisfied.

The locally summable functions $e_{+}^{x}$ and $e_{-}^{x}$ are defined by

$$
e_{+}^{x}=H(x) e^{x} \quad e_{-}^{x}=H(-x) e^{x} .
$$

In the following we need the following lemma, which is easily proved by induction.

\section{Lemma 1.}

$$
\begin{aligned}
\int_{0}^{u} t^{k} e^{-t} \mathrm{~d} t & =-\sum_{i=0}^{k} \frac{k !}{i !} u^{i} e^{-u}+k ! \\
\int_{0}^{u} t^{k} e^{-2 t} \mathrm{~d} t & =-\sum_{i=0}^{k} \frac{k !}{2^{k-i+1} i !} u^{i} e^{-2 u}+\frac{k !}{2^{k+1}},
\end{aligned}
$$

for $k=0,1,2, \ldots$

We now prove the following theorem. 
Theorem 1. The convolution $x^{r} \mathrm{ei}_{+}(x) * x^{s} e_{+}^{x}$ exists and

$$
\begin{aligned}
x^{r} \mathrm{ei}_{+}(x) * x^{s} e_{+}^{x}= & \\
= & \sum_{k=0}^{s} \sum_{i=1}^{r+k}\left(\begin{array}{l}
s \\
k
\end{array}\right)(-1)^{k}(r+k) ! x^{s-k}\left[\sum_{j=0}^{i-1} \frac{(i-1) !}{2^{i-j} i ! j !} x^{j} e_{+}^{-x}-\frac{(i-1) !}{2^{i} i !} e_{+}^{x}\right] \\
& +\sum_{k=0}^{s}\left(\begin{array}{l}
s \\
k
\end{array}\right)(-1)^{k}(r+k) ! x^{s-k}\left[e^{x} \mathrm{ei}_{+}(2 x)-e^{x} \mathrm{ei}_{+}(x)+\ln 2 e_{+}^{x}\right] \\
& -\sum_{k=0}^{s}\left(\begin{array}{l}
s \\
k
\end{array}\right)(-1)^{k}(r+k) ! x^{s-k}\left[\sum_{i=1}^{r+k} \frac{x^{i}}{i !}+\left(1-e^{x}\right)\right] \mathrm{e} \mathrm{i}_{+}(x),
\end{aligned}
$$

for $r, s=0,1,2, \ldots$ and $r, s$ not both zero.

In particular,

$$
\begin{aligned}
x^{r} \mathrm{e} \mathrm{i}_{+}(x) * e_{+}^{x}=r ! & \sum_{i=1}^{r}\left[\sum_{j=0}^{i-1} \frac{(i-1) !}{2^{i-j} i ! j !} x^{j} e_{+}^{-x}-\frac{(i-1) !}{2^{i} i !} e_{+}^{x}\right] \\
+ & r !\left[e^{x} \mathrm{ei}_{+}(2 x)-e^{x} \mathrm{ei}_{+}(x)+\ln 2 e_{+}^{x}\right] \\
& -r !\left[\sum_{i=1}^{r} \frac{x^{i}}{i !}+\left(1-e^{x}\right)\right] \mathrm{ei}_{+}(x),
\end{aligned}
$$

for $r=1,2, \ldots$ and

$$
\mathrm{ei}_{+}(x) * e_{+}^{x}=-\mathrm{ei}_{+}(x)+e^{x} \mathrm{ei}_{+}(2 x)+\ln 2 e_{+}^{x} .
$$

Proof. The convolution $x^{r} \mathrm{ei}_{+}(x) * x^{s} e_{+}^{x}=0$ if $x<0$ and so when $x>0$, we have

$$
\begin{aligned}
x^{r} \mathrm{ei}_{+}(x) * x^{s} e_{+}^{x}= & \int_{0}^{x} t^{r}(x-t)^{s} e^{x-t} \int_{t}^{\infty} u^{-1} e^{-u} \mathrm{~d} u \mathrm{~d} t \\
= & \int_{0}^{x} u^{-1} e^{x-u} \int_{0}^{u} t^{r}(x-t)^{s} e^{-t} \mathrm{~d} t \mathrm{~d} u \\
& \quad+\int_{x}^{\infty} u^{-1} e^{x-u} \int_{0}^{x} t^{r}(x-t)^{s} e^{-t} \mathrm{~d} t \mathrm{~d} u \\
= & I_{1}+I_{2},
\end{aligned}
$$

where

$$
\begin{aligned}
\int_{0}^{u} t^{r} & (x-t)^{s} e^{-t} \mathrm{~d} t= \\
& =\sum_{k=0}^{s}\left(\begin{array}{l}
s \\
k
\end{array}\right)(-1)^{k} x^{s-k} \int_{0}^{u} t^{r+k} e^{-t} \mathrm{~d} t \\
& =-\sum_{k=0}^{s}\left(\begin{array}{l}
s \\
k
\end{array}\right)(-1)^{k}(r+k) ! x^{s-k}\left[\sum_{i=1}^{r+k} \frac{u^{i}}{i !} e^{-u}+\left(e^{-u}-1\right)\right]
\end{aligned}
$$


and in particular when $r=s=0$,

$$
\int_{0}^{u} e^{-t} \mathrm{~d} t=-e^{-u}+1
$$

on using the lemma.

Hence

$$
\begin{aligned}
I_{1}=- & \sum_{k=0}^{s}\left(\begin{array}{l}
s \\
k
\end{array}\right)(-1)^{k}(r+k) ! x^{s-k} e^{x} . \\
& \cdot \int_{0}^{x}\left[\sum_{i=1}^{r+k} \frac{u^{i-1}}{i !} e^{-2 u}+u^{-1}\left(e^{-2 u}-e^{-u}\right)\right] \mathrm{d} u \\
=- & \sum_{k=0}^{s}(r+k) ! \sum_{i=1}^{r+k}\left(\begin{array}{l}
s \\
k
\end{array}\right)(-1)^{k} x^{s-k} e^{x} \int_{0}^{x} \frac{u^{i-1}}{i !} e^{-2 u} \mathrm{~d} u \\
& -\sum_{k=0}^{s}\left(\begin{array}{l}
s \\
k
\end{array}\right)(-1)^{k}(r+k) ! x^{s-k} e^{x} \int_{0}^{x} u^{-1}\left(e^{-2 u}-e^{-u}\right) \mathrm{d} u .
\end{aligned}
$$

Further, we have

$$
\int_{0}^{x} \frac{u^{i-1}}{i !} e^{-2 u} \mathrm{~d} u=-\sum_{j=0}^{i-1} \frac{(i-1) !}{2^{i-j} i ! j !} x^{j} e^{-2 x}+\frac{(i-1) !}{2^{i} i !},
$$

on using the lemma, and

$$
\begin{aligned}
\int_{0}^{x} & u^{-1}\left[e^{-u}-H(1-u)\right] \mathrm{d} u= \\
= & \int_{0}^{\infty} u^{-1}\left[e^{-u}-H(1-u)\right] \mathrm{d} u \\
& \quad-\int_{x}^{\infty} u^{-1} e^{-u} \mathrm{~d} u+\int_{x}^{\infty} u^{-1} H(1-u) \mathrm{d} u \\
= & -\gamma-\mathrm{ei}_{+}(x)+\int_{x}^{\infty} u^{-1} H(1-u) \mathrm{d} u .
\end{aligned}
$$

Similarly

$$
\int_{0}^{x} u^{-1}\left[e^{-2 u}-H(1-2 u)\right] \mathrm{d} u=-\gamma-\mathrm{ei}_{+}(2 x)+\int_{x}^{\infty} u^{-1} H[1-2 u] \mathrm{d} u .
$$

It follows from equations (21) and (22) that

$$
\begin{aligned}
& \int_{0}^{x} u^{-1}\left(e^{-u}-e^{-2 u}\right) \mathrm{d} u= \\
& \quad=\mathrm{ei}_{+}(2 x)-\mathrm{ei}_{+}(x)+\int_{0}^{\infty} u^{-1}[H(1-u)-H(1-2 u)] \mathrm{d} u \\
& \quad=\mathrm{ei} i_{+}(2 x)-\mathrm{ei}_{+}(x)+\ln 2 .
\end{aligned}
$$


In particular, when $r=s=0$, we have

$$
I_{1}=\left[\mathrm{ei}_{+}(2 x)-\mathrm{ei}_{+}(x)+\ln 2\right] e_{+}^{x} .
$$

Next, as in equation (17), we have

$$
\begin{aligned}
\int_{0}^{x} t^{r}(x-t)^{s} e^{-t} \mathrm{~d} t & = \\
& =-\sum_{k=0}^{s}\left(\begin{array}{l}
s \\
k
\end{array}\right)(-1)^{k}(r+k) ! x^{s-k}\left[\sum_{i=1}^{r+k} \frac{x^{i}}{i !} e^{-x}+\left(e^{-x}-1\right)\right]
\end{aligned}
$$

and so

$$
I_{2}=-\sum_{k=0}^{s}\left(\begin{array}{l}
s \\
k
\end{array}\right)(-1)^{k}(r+k) ! x^{s-k}\left[\sum_{i=1}^{r+k} \frac{x^{i}}{i !}+\left(1-e^{x}\right)\right] \mathrm{ei}_{+}(x) .
$$

In particular, when $r=s=0$, we have

$$
I_{2}=\left(e^{x}-1\right) \int_{x}^{\infty} u^{-1} e^{-u} \mathrm{~d} u=\left(e^{x}-1\right) \mathrm{ei}_{+}(x) .
$$

Equation (13) now follows from equations (20), (21), (22), (25) and (26).

Equation (14) follows on putting $s=0$ in equation (13) and equation (15) follows on putting $r=0$ in equation (14).

In the corollary, the distribution $x_{+}^{-2}$ is defined by $x_{+}^{-2}=\left(x_{+}^{-1}\right)^{\prime}$ and not as in Gel'fand and Shilov.

Corollary 1.1. The convolutions $\left(e^{-x} x_{+}^{-1}\right) * e_{+}^{x}$ and $\left(e^{-x} x_{+}^{-2}\right) * e_{+}^{x}$ exist and

$$
\begin{aligned}
& \left(e^{-x} x_{+}^{-1}\right) * e_{+}^{x}=-e^{x} \mathrm{ei}_{+}(2 x)-\gamma(2) e_{+}^{x} \\
& \left(e^{-x} x_{+}^{-2}\right) * e_{+}^{x}=2 e^{x} \mathrm{ei}_{+}(2 x)+2 \gamma(2) e_{+}^{x}-e^{-x} x_{+}^{-1} .
\end{aligned}
$$

Proof. The convolution $\left(e^{-x} x_{+}^{-1}\right) * e_{+}^{x}$ exists by Definition 2 , since $e^{-x} x_{+}^{-1}$ and $e_{+}^{x}$ are both bounded on the left. From equation (12), we have

$$
\begin{aligned}
{\left[\mathrm{ei}_{+}(x) * e_{+}^{x}\right]^{\prime} } & =-\left[e^{-x} x_{+}^{-1}+\gamma \delta(x)\right] * e_{+}^{x} \\
& =-\left(e^{-x} x_{+}^{-1}\right) * e_{+}^{x}-\gamma e_{+}^{x} \\
& =\mathrm{ei}_{+}(x) *\left[e_{+}^{x}+\delta(x)\right] \\
& =e^{x} \mathrm{ei}_{+}(2 x)+\ln 2 e_{+}^{x}
\end{aligned}
$$

and equation (28) follows.

From equations (12) and (28), we now have

$$
\begin{aligned}
{\left[\left(e^{-x} x_{+}^{-1}\right) * e_{+}^{x}\right]^{\prime} } & =-\left(e^{-x} x_{+}^{-1}+e^{-x} x_{+}^{-2}\right) * e_{+}^{x} \\
& =e^{x} \mathrm{ei}_{+}(2 x)+\gamma(2) e_{+}^{x}-\left(e^{-x} x_{+}^{-2}\right) * e_{+}^{x} \\
& =\left(e^{-x} x_{+}^{-1}\right) *\left[e_{+}^{x}+\delta(x)\right] \\
& =-e^{x} \mathrm{ei}_{+}(2 x)-\gamma(2) e_{+}^{x}+e^{-x} x_{+}^{-1}
\end{aligned}
$$


and equation (29) follows.

Theorem 2. The convolution $x^{r} \mathrm{ei}_{+}(x) * x^{s} e^{x}$ exists and

$$
\begin{aligned}
x^{r} \mathrm{ei}_{+}(x) * x^{s} e^{x}=- & \sum_{k=0}^{s} \sum_{i=1}^{r+k}\left(\begin{array}{l}
s \\
k
\end{array}\right) \frac{(-1)^{k}(r+k) !}{2^{i} i !} x^{s-k} e^{x} \\
& +\sum_{k=0}^{s}\left(\begin{array}{l}
s \\
k
\end{array}\right)(-1)^{k} \ln 2(r+k) ! x^{s-k} e^{x},
\end{aligned}
$$

for $r, s=0,1,2, \ldots$ and $r, s$ not both zero.

In particular

$$
x^{r} \mathrm{ei}_{+}(x) * e^{x}=-\sum_{i=1}^{r} \frac{r !}{2^{i} i !} e^{x}+\ln 2 r ! e^{x},
$$

for $r=1,2, \ldots$ and

$$
\begin{aligned}
\mathrm{ei}_{+}(x) * e^{x} & =\ln 2 e^{x} \\
\mathrm{ei}_{+}(x) * x e^{x} & =\ln 2 x e^{x}-\ln 2 e^{x}+\frac{1}{2} e^{x}
\end{aligned}
$$

Proof. We have

$$
\begin{aligned}
x^{r} \mathrm{ei}_{+}(x) * x^{s} e^{x}= & \int_{0}^{\infty} t^{r}(x-t)^{s} e^{x-t} \int_{t}^{\infty} u^{-1} e^{-u} \mathrm{~d} u \mathrm{~d} t \\
= & \int_{0}^{\infty} u^{-1} e^{x-u} \int_{0}^{u} t^{r}(x-t)^{s} e^{-t} \mathrm{~d} t \mathrm{~d} u \\
= & -\sum_{k=0}^{s}\left(\begin{array}{l}
s \\
k
\end{array}\right)(-1)^{k}(r+k) ! x^{s-k} e^{x} \sum_{i=1}^{r+k} \int_{0}^{\infty} \frac{u^{i-1}}{i !} e^{-2 u} \mathrm{~d} u \\
& -\sum_{k=0}^{s}\left(\begin{array}{l}
s \\
k
\end{array}\right)(-1)^{k}(r+k) ! x^{s-k} e^{x} \int_{0}^{\infty}\left(u^{-1} e^{-2 u}-u^{-1} e^{-u}\right) \mathrm{d} u \\
= & -\sum_{k=0}^{s} \sum_{i=1}^{r+k}\left(\begin{array}{l}
s \\
k
\end{array}\right) \frac{(-1)^{k}(r+k) !}{2^{i} i} x^{s-k} e^{x} \\
& +\sum_{k=0}^{s}\left(\begin{array}{l}
s \\
k
\end{array}\right)(-1)^{k} \ln 2(r+k) ! x^{s-k} e^{x}
\end{aligned}
$$

on making use of equation (17), the lemma and noting that

$$
\begin{aligned}
\int_{0}^{\infty} u^{-1}\left(e^{-2 u}-e^{-u}\right) \mathrm{d} u & =\int_{0}^{\infty} \ln u\left(2 e^{-2 u}-e^{-u}\right) \mathrm{d} u \\
& =\Gamma^{\prime}(1)-\ln 2-\Gamma^{\prime}(1)=-\ln 2,
\end{aligned}
$$

proving equation (30).

Equation (31) follows on putting $s=0$ in equation (30) and equation (32) follows on putting $r=0$ in equation (31). 
Equation (31) follows on putting $r=0$ and $s=1$ in equation (30).

Corollary 2.1. The convolution $\left(e^{-x} x_{+}^{-n}\right) * e^{x}$ exists and

$$
e^{-x} x_{+}^{-n} * e^{x}=\frac{(-1)^{n} 2^{n-1}}{(n-1) !} \gamma(2) e^{x}
$$

for $n=1,2, \ldots$

In particular,

$$
e^{-x} x_{+}^{-1} * x e^{x}=-\gamma(2) x e^{x}-\frac{1}{2} e^{x} .
$$

Proof. Differentiating equation (32), we get

$$
\left[-e^{-x} x_{+}^{-1}-\gamma \delta(x)\right] * e^{x}=-\left(e^{-x} x_{+}^{-1}\right) * e^{x}-\gamma e^{x}=\ln 2 e^{x}
$$

and we see that equation (34) is true when $n=1$.

Now assume that equation (34) is true for some $n$. Then differentiating equation (34), we get

$$
\left(-e^{-x} x_{+}^{-n}-n e^{-x} x_{+}^{-n-1}\right) * e^{x}=\left(e^{-x} x_{+}^{-n}\right) * e^{x} .
$$

It follows that

$$
\begin{aligned}
n e^{-x} x_{+}^{-n-1} * e^{x} & =-2\left(e^{-x} x_{+}^{-n}\right) * e^{x} \\
& =\frac{(-1)^{n+1} 2^{n}}{(n-1) !} \gamma(2) e^{x}
\end{aligned}
$$

and so equation (33) is true for $n+1$. Equation (34) now follows by induction.

Differentiating equation (33), we get

$$
\left[-e^{-x} x_{+}^{-1}-\gamma \delta(x)\right] * x e^{x}=\ln 2 x e^{x}+\frac{1}{2} e^{x}
$$

and equation (35) follows.

For further results involving the exponential integral, see $[2,3,4,5]$ and [6].

\section{REFERENCES}

[1] B. Fisher and J.D. Nicholas, The exponential integral and the convolution, SUT J. Math., 33(2)(1997), 139-148.

[2] B. Fisher and J.D. Nicholas, The neutrix convolution product of ei $(\lambda x)$ and $x^{r}$, Math. Montisnigri, 9(1998), 51-64.

[3] B. Fisher and J.D Nicholas, On the exponential integral, Hacet. Bull. Nat. Sci. Eng. Ser. B, 28(1999), 55-64.

[4] B. Fisher and J.D Nicholas, The exponential integral and the neutrix convolution product, J. Nat. Sci. Math., 40(1,2)(2000), 23-36.

[5] B. Fisher and J.D. Nicholas, On the exponential integral and the non-commutative convolution product, Integral Transforms Spec. Funct., 11(4)(2001), 353-362. 
[6] B. Fisher, E. Özçag and Ü. Gülen, The exponential integral and the commutative neutrix convolution product, J. Analysis, 7(1999), 7-20.

[7] I.M. Gel'fand and G.E. Shilov, Generalized functions, Vol. I, Academic Press (1964).

[8] I.N. Sneddon, Special Functions of Mathematical Physics and Chemistry, Oliver and Boyd, (1961).

\section{Brian Fisher}

Department of Mathematics

UNIVERSITY OF LEICESTER

LEICESTER, LE1 7RH

ENGLAND

E-mail address: fbr@le.ac.uk

\section{Fatma Al-Sirehy}

Department of Mathematics

King Abdulaziz University

JEDDAH

SAudi Arabia

E-mail address: falserehi@kau.edu.sa 